\title{
Impactos ambientales asociados con el proceso de producción del concreto
}

\section{(Aspects and environmental impacts associated with the production of concrete)}

\author{
Aura Navas de García ${ }^{1}$, Rosa E. Reyes Gil ${ }^{1,2}$ y Luis E. Galván Rico ${ }^{1,3}$
}

\begin{abstract}
Resumen:
Las Plantas de Concreto son importantes para la economía de un país. Sin embargo, este tipo de industria ocasiona importantes impactos ambientales en cada uno de los pasos involucrados en el proceso industrial de producción del concreto los cuales son analizados en esta investigación. Adicionalmente, se evaluó la percepción de los expertos y los trabajadores en el área, respecto a los impactos ambientales asociados con la actividad de una empresa productora de concreto. La metodología aplicada para el análisis de pasos del proceso de producción fue de tipo documental en tanto que la utilizada para evaluar la percepción de los impactos ambientales fue descriptiva de campo de corte transversal, aplicando entrevistas a los trabajadores relacionados con el proceso de fabricación del concreto. Entre los principales impactos ambientales identificados se encuentran las emisiones de polvo y el manejo inadecuado de efluentes, materiales peligrosos y no peligrosos. Entre las propuestas presentadas para enfrentar estos impactos están: instalación de colectores de polvo, fosas de sedimentación, planes de manejo de materiales peligrosos y no peligrosos, entre otras. Del análisis de los resultados obtenidos se propone la incorporación de los principios de Responsabilidad Social Empresarial para la mejora o minimización de los impactos adversos detectados.
\end{abstract}

Palabras clave: Plantas de concreto; aspectos ambentales; ciclo de vida; responsabilidad social empresarial.

\begin{abstract}
:
Concrete plants are important to the economy of a country. However, this industry causes major environmental impacts in each of the steps involved in the manufacturing process of concrete production which are analyzed in this research. In addition, the perception of experts and workers in the area, about the environmental impacts associated with the activity of a producer of concrete was evaluated. The methodology used for the analysis of steps in the production process was as kind documentary. For to evaluate perception of environmental impacts the methodology used was descriptive non experimental, using interviews with workers related to the manufacturing process of the concrete. Among the major identified environmental impacts are dust emissions and improper handling of effluents, hazardous and non-hazardous materials. Among the proposals put forward to address these impacts include: installation of dust collectors, settling tanks, management plans for hazardous and non-hazardous materials, among others. An analysis of the results incorporating the principles of corporate social responsibility for improving or minimizing adverse impacts are detected proposed.
\end{abstract}

Keywords: Concrete plants; environmental aspects; life cycle; corporate social responsibility.

\footnotetext{
${ }^{1}$ Universidad Simón Bolívar, Caracas - Venezuela (rereyes@usb.ve)

${ }^{2}$ Universidad de Guayaquil, Guayaquil - Ecuador

${ }^{3}$ Instituto Tecnológico Superior Simón Bolívar, Guayaquil - Ecuador (Igalvan@itssb.edu.ec)
} 


\section{Introducción}

La producción de concreto premezclado es una actividad industrial fundamental para la implantación de proyectos de construcción en general, y como tal, está vinculada al crecimiento económico del país. Sin embargo, cuando esta actividad no cuenta con una gestión ambiental bien concebida y eficazmente llevada a la práctica, se ocasionan impactos significativos al ambiente debido al uso intensivo de energía y materia prima (minerales y agua), generando desechos, emisiones y efluentes contaminantes (Prolys, 2008). En efecto, las Plantas de Concreto Premezclado se encuentran entre las obras, cuyo establecimiento inevitablemente genera alteraciones del ambiente donde se localizan (Fonseca y Reyes, 2015).

Desde el punto de vista de la legislación ambiental vigente en la mayoría de los países, estas plantas son consideradas como actividades susceptibles de degradar el ambiente y requieren de la presentación de un Estudio de Impacto Ambiental manera obligatoria. En este contexto, la elaboración del concreto premezclado debe considerar exigencias legales y técnicas que permitan garantizar un desempeño ambiental responsable en el marco del Desarrollo Sustentable (Gabaldón, 2006).

Desde el punto de vista empresarial, el sector industrial ha percibido que los aspectos ambientales bien administrados pueden generar valor económico corporativo, bien sea por la reducción del riesgo, la reducción de los costos totales de producción, el aumento de los ingresos y la buena imagen pública de la empresa (Clemente et al., 2005). En este sentido, la creciente preocupación de la opinión pública por los problemas ambientales ha obligado a muchas empresas a adoptar procedimientos de mejora en sus procesos de producción y a buscar soluciones a los problemas generados por sus actividades empresariales. Estas soluciones se han manifestado a través de diversas líneas de acción, entre las que destacan: el establecimiento de controles operativos, el desarrollo de políticas públicas y legislaciones en materia ambiental que buscan resguardar el ambiente mundial a través de la difusión de campañas formativas e informativas, el establecimiento de programas de educación ambiental y la adopción de herramientas de gestión ambiental por parte de las empresas (Reyes et al., 2002, 2003; Guédez et al., 2003; Villegas et al., 2004,2005; Galván et al., 2009, 2012).

Con base en estas consideraciones, el presente trabajo tiene como objetivo diagnosticar la situación actual de una empresa de concreto en su entorno ambiental y formular propuestas para su mejora. Para ello se analizaron las diferentes etapas del proceso de producción del concreto premezclado para determinar los problemas ambientales generados en cada etapa y proponer soluciones. También se indagó la percepción de los trabajadores de la empresa en relación a los problemas existentes con su entorno ambiental. 


\section{Metodología}

El presente estudio fue desarrollado en una Planta de Concreto ubicada en el área central de Venezuela, encargada de la fabricación y venta de concreto premezclado, con una capacidad instalada de producción de $96.000 \mathrm{~m}^{3} /$ año y cuyas instalaciones están ubicadas en un área aproximada de $14.000 \mathrm{~m}^{2}$ de superficie.

La investigación se realizó en dos etapas:

1. Levantamiento de la información documental, a través de la revisión bibliográfica de documentos y publicaciones para conocer con detalle el proceso de producción, los impactos ambientales asociados y las medidas propuestas (García, 2004; Galán, 2010; O'Reilly et al., 2010; Fonseca y Reyes, 2015).

2. Visitas in situ a la planta de concreto premezclado para la constatación de la información documental y la aplicación de las entrevistas para determinar la percepción de los trabajadores. En esta etapa, la investigación es descriptiva de corte transversal por cuanto se analiza la situación actual de la planta de concreto sin manipular variable alguna y la información es colectada solo una vez (Hernández et al., 2010; Arias, 2012).

La entrevista aplicada fue abierta semiestructurada, probada y validada por expertos, a la que posteriormente se le aplicó un análisis de contenido. Las entrevistas se aplicaron a una muestra representativa de 15 trabajadores, con un tiempo promedio de trabajo de 5 años en la planta de concreto, con disposición para suministrar información e involucrados con los aspectos de producción.

\section{Resultados y Discusión}

\subsection{Análisis de las etapas del proceso de producción del concreto premezclado. Impactos ambientales}

La Figura 1 muestra el proceso de producción del concreto premezclado incluyendo las entradas y salidas en cada etapa del proceso.

Etapa 1. Recepción de la materia prima. La materia prima para la producción del concreto está constituida por arena, piedra, agua, cemento y aditivos. Cada uno de ellos posee un origen distinto. La arena o agregado fino está conformado por arena natural o arena obtenida por trituración. La piedra o agregado grueso, está constituida por rocas provenientes de la trituración y de río, los orígenes más comunes son caliza, gneis y silicio. El cemento es un material polvoriento que al mezclarse con agua, forma una pasta que es capaz de fraguar y endurecerse, se le conoce también como conglomerante hidráulico. Los aditivos son productos químicos, líquidos o sólidos, que se pueden agregar a la mezcla del concreto antes o durante el mezclado; el uso de aditivos ayuda a modificar las propiedades del concreto tanto en estado fresco como endurecido. 


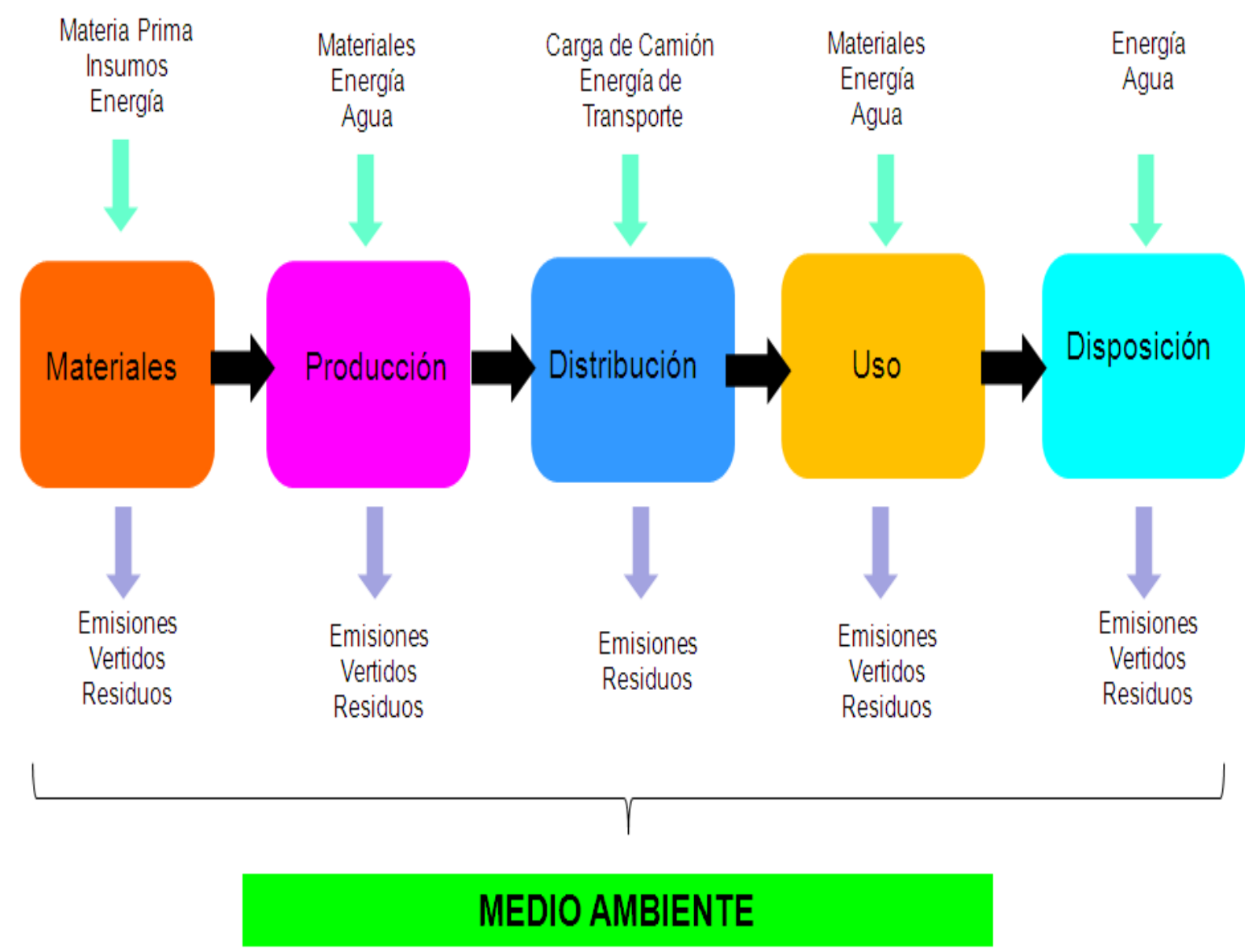

Figura 1. Proceso de producción del concreto premezclado

Debe considerarse la energía y el agua como insumos imprescindibles para el proceso de producción del concreto. La energía eléctrica es proporcionada por la empresa nacional proveedora de electricidad permitiendo la movilización de los equipos de las Plantas y el funcionamiento de las áreas administrativas. Otra forma de energía utilizada es la obtenida a partir de combustibles fósiles como el gasoil para los camiones trompos y retroexcavadoras, entre otros. El agua juega un papel fundamental a lo largo del todo el proceso de la elaboración del concreto. En este caso de estudio, proviene de la red de suministro público. El agua a ser utilizada en este proceso de producción industrial debe cumplir con las Normas COVENIN 2385:2000.

El principal impacto ambiental generado en esta etapa es la generación de emisiones de polvo provenientes del manejo de los agregados en el punto de descarga, acarreo y consumo en la Planta. Para ello se ejecutan algunas medidas de control, tales como:

1. Colocar lonas sobre la carga de los camiones.

2. Para el caso de la piedra de $3 / 4 "$, se realiza el riego mediante aspersores. 
Los impactos ambientales causados por el cemento se encuentran presentes a lo largo del proceso de producción del concreto, por ello se propones las siguientes acciones:

1. Disposición en silos para su adecuado almacenamiento con un colector de polvo para la captura de partículas de cemento.

2. Utilización del equipo que actúa como aspiradora del cemento (drybatch) que se dispersa en el punto de descarga cuando el material es depositado en el camión.

3. El cemento también se encuentra presente en la lechada de cemento; la retención de este material ocurre en la fosa de decantación. Cuando se realiza el mantenimiento de esta fosa el material depositado es considerado como escombro y dispuesto en los vertederos.

Etapa 2. Producción del concreto. El concreto es el material resultante de la mezcla del cemento con agregados de diferente tamaño (grava, gravilla y arena) y agua. El cemento, mezclado con el agua y los agregados, se convierte en una pasta moldeable con propiedades adherentes, que en pocas horas fragua y se endurece tornándose de la forma del recipiente que lo contiene.

El proceso de producción de concreto en el área de estudio sigue los siguientes pasos:

1. El conductor de la unidad mezcladora coloca el camión en la zona de carga y acelera el trompo mezclador a una velocidad aproximada de $15-20$ revoluciones $/ \mathrm{min}$.

2. Se descarga un porcentaje de agua correspondiente a la capacidad del camión.

3. También, junto con el agua, se descarga la cantidad de aditivo correspondiente por cada bacheo.

4. Se abre la jaiba de la báscula de agregados, y comienza a adicionarse la piedra y la arena simultáneamente junto con la descarga del cemento.

5. Finalizada la descarga de cemento y agregados, se añade el resto del agua según el diseño. Esto ayuda a limpiar el embudo de la tolva del camión de restos de cemento y agregados.

6. Al finalizar la mezcla, el conductor de la Unidad Mezcladora reduce la velocidad del trompo a velocidad de agitación y da inicio al proceso de Distribución del Concreto Premezclado. La correcta mezcla se realiza en aproximadamente 70 revoluciones del trompo mezclador del camión, es decir, en 3,5 minutos.

Este proceso es controlado por el sistema automático o mediante la Consola Manual, aparte de la intervención del conductor de la Unidad Mezcladora en el manejo y control del trompo mezclador de los camiones. 
Los principales impactos ambientales en esta etapa son:

1. Afectación de la calidad del aire por la generación de polvos y gases. Se propone el diseño y puesta en marcha de un Programa de prevención y control de emisiones atmosféricas durante la operación de la planta en función de la legislación ambiental vigente (Decreto № 638 relativo a Normas sobre calidad del aire y control de la contaminación atmosférica y Decreto $N^{\circ} 2.673$ relativo a las Normas sobre emisiones de fuentes móviles).

2. Incremento de los niveles de ruido en la planta de concreto y áreas cercanas. Se propone el diseño y puesta en ejecución de un Programa de prevención y control de ruido durante la etapa de operación de la planta para el cumplimiento de la legislación vigente (Decreto № 2.217 relativo a las Normas sobre el control de la contaminación generada por ruido).

3. Afectación de la calidad de las aguas por descargas de efluentes parcialmente tratados en la etapa de operación de la planta. Se propone la implantación de los controles necesarios, con el fin de realizar el adecuado manejo de los efluentes producto de la operación de la planta de concreto, para evitar con ello problemas de contaminación de las aguas y suelos, sobre todo en el caso de aquellos de tipo peligroso. Asimismo, cumplir con la normativa vigente sobre control de la calidad de agua subterránea establecida en la Ley de aguas (GO N³8.595) y el Decreto № 883 relativo a la Normas para la clasificación y el control de la calidad de los cuerpos de agua y vertidos o efluentes líquidos.

4. Manejo inadecuado de desechos peligrosos y no peligrosos en la planta, el taller de mantenimiento mecánico y áreas cercanas. Se propone el diseño y puesta en práctica de un Plan de manejo de los desechos no peligrosos y peligrosos generados en la etapa de operación de la planta para el cumplimiento de la normativa correspondiente (Decreto № 2.216 sobre Normas para el manejo de desechos sólidos de origen doméstico, comercial, industrial o de cualquier otra naturaleza que no sean peligrosos, Decreto № 2.635 sobre Normas para el control de la recuperación de materiales peligrosos y el manejo de desechos peligrosos, Ley sobre sustancias, materiales y desechos peligrosos, GO № 5.554 y Ley de gestión integral de la basura, GO № 6.071).

Etapa 3. Uso del producto. Los productos de concreto son variados y numerosos; y se pueden clasificar en dos grandes grupos:

- Los hormigones premezclados y morteros pre-dosificados (productos intermedios).

- Productos prefabricados de hormigón.

La diferencia entre estos dos grupos de productos es que el primero de ellos, luego de realizada la mezcla, se despacha directamente a la obra de construcción, donde es usado para hacer productos in situ. Los prefabricados, en cambio, son elaborados dentro de la fábrica, donde se 
almacenan, para luego ser comercializados como productos determinados (CONAMA, 2001). La durabilidad del concreto es un atributo sustentable debido a que no se oxida, no se pudre y no se quema, al mismo tiempo que se requiere menos energía y recursos a lo largo del tiempo para reparar o reemplazar edificaciones hechas con concreto.

Por otro lado, el concreto es el material de construcción más utilizado actualmente en el mundo entero, las estructuras de concreto han pasado la prueba del tiempo por más de 200 años. Debido a su longevidad, su uso es una solución de diseño viable para el desarrollo sustentable. Las estructuras ejecutadas con concreto tienen un desempeño óptimo en cuanto al consumo de energía. En efecto, las edificaciones hechas con paredes de concreto no están sujetas a altas fluctuaciones diarias de temperatura, lo que se traduce en una reducción de aproximadamente del $25 \%$ en el consumo de calefacción y aire acondicionado y por lo tanto en menores costos. De igual forma, los equipos de ventilación, calefacción y aire acondicionado pueden diseñarse para capacidades menores.

Etapa 4. Disposición final. Cuando este concreto no puede ser colocado en las diferentes obras de construcción puede tener distintas opciones de uso tendientes a la minimización, reciclaje y recuperación (CONAMA; 2001). Entre los posibles usos del concreto al final de su ciclo de vida se encuentran:

1.Como relleno y recuperación de terrenos y para construcción de terraplenes.

2.Reciclaje para utilizar en el asfalto

3.Como sub-bases de carreteras

4.El concreto pobre se puede utilizar para hacer molde para pisos.

Lo más sencillo es utilizar los desechos como relleno de escombros. En el caso de su empleo como sub-base o base de carreteras deben ser molidos adecuadamente y cumplir con los requerimientos para este uso.

La Tabla 1 muestra los principales impactos ambientales asociados con las etapas del proceso de producción de concreto premezclado.

\subsection{Percepción de los trabajadores sobre los problemas en su entorno ambiental}

\section{Conocimiento del proceso de producción.}

Los trabajadores entrevistados ( $57 \%$ ) tienen más de 10 años en la compañía, lo cual permite inferir que conocen bien la acción de la empresa en relación con la problemática ambiental y las mejoras implementadas. El $64 \%$ de la muestra seleccionada trabaja en el área operativa de la Planta de Concreto, lo cual permite suponer que estos trabajadores se hallan totalmente involucrados con el proceso de producción y lo conocen integralmente. Todos los trabajadores 
entrevistados conocen el proceso de elaboración de concreto y por ende, los insumos de entrada, el proceso propiamente dicho y el producto final, sus características físicas, aplicaciones y usos.

Tabla 1. Impactos ambientales asociados con cada etapa del proceso productivo del concreto premezclado

\begin{tabular}{|c|c|}
\hline ETAPA DEL PROCESO & IMPACTOS AMBIENTALES ASOCIADOS \\
\hline 1. Recepción de la materia prima & $\begin{array}{l}\text { - Contaminación atmosférica: generación de emisiones } \\
\text { de polvo. } \\
\text { - } \quad \text { Abundante uso de agua y energía. } \\
\text { - } \text { sisposición en fosas de decantación que requieren } \\
\text { saneanto y disposición del material solidificado. }\end{array}$ \\
\hline 2.Producción del concreto & $\begin{array}{l}\text { - Afectación de la calidad del aire: generación de } \\
\text { polvos y gases. } \\
\text { - } \text { Incremento de los niveles de ruido en la planta de } \\
\text { concreto y áreas cercanas. } \\
\text { - Afectación de la calidad de las aguas por descargas } \\
\text { de efluentes parcialmente tratados durante la } \\
\text { operación de la planta. } \\
\text { Manejo inadecuado de desechos peligrosos y no } \\
\text { peligrosos en la planta y áreas cercanas. }\end{array}$ \\
\hline 3.Uso del producto & $\begin{array}{l}\text { - Afectación de la calidad de las aguas como } \\
\text { consecuencia del proceso de lavado y mantenimiento } \\
\text { de las diversas obras. } \\
\text { - Manejo inadecuado de desechos sólidos en obras de } \\
\text { remodelación. }\end{array}$ \\
\hline 4.Disposición final & $\begin{array}{l}\text { - Disposición inadecuada de los desechos generados } \\
\text { en las obras de demolición. } \\
\text { - Incremento en los niveles de ruido durante las obras } \\
\text { de desmantelamiento y demolición. } \\
\text { - Contaminación atmosférica por generación de polvo y } \\
\text { gases. } \\
\text { Afectación a la calidad de las aguas por generación } \\
\text { de efluentes contaminados. }\end{array}$ \\
\hline
\end{tabular}

\section{Percepción de los problemas ambientales y soluciones propuestas.}

La contaminación del agua y del aire fueron seleccionados como los mayores problemas ambientales que los trabajadores perciben en el proceso de la fabricación del concreto. El agua se utiliza a lo largo del todo el proceso de elaboración de concreto, ya que es un recurso fundamental para la mezcla de la materia prima, adicionalmente se emplea para el lavado de camiones, en la etapa de muestreo y lavado de patios para el control de las emisiones de polvo fugitivas. En el caso del aire, las emisiones de polvo son evidentes para los trabajadores y espectadores casuales de la Planta de Concreto, debido al bombeo del cemento hacia los silos (área de almacenamiento), acarreo de la piedra hasta la tolva y por el paso constante de los vehículos pesados y livianos. El caso de la contaminación de los suelos, no es tan evidente para los trabajadores porque ésta está referida a casos puntuales por la manipulación inadecuada de los materiales peligrosos recuperables y desechos peligrosos. Sin embargo, en el caso de materiales no peligrosos de tipo industrial como los escombros, se manejan grandes volúmenes generados por el mantenimiento de la fosa de lavado y los cilindros o probetas de muestreo. 
Los trabajadores señalaron un conjunto de alternativas para detectar y solventar los problemas ambientales que ocasionan las Plantas de Concreto en su entorno inmediato. Entre ellas se encuentran: el uso de aspersores, la instalación de plantas recicladoras, el mantenimiento periódico de los filtros, la realización de Estudios de Calidad del Aire, el mantenimiento de las fosas de lavado, el confinamiento de los agregados y la pavimentación del área del proceso de producción, y la dotación de equipos de protección personal.

\section{Actividades ambientales y participación}

Las campañas ambientales son realizadas por las empresas para moldear el comportamiento ambiental de su personal y construir una imagen corporativa respetuosa con el ambiente. Estas campañas ambientales deberían promover cambios en el comportamiento que pueden llegar hasta el entorno familiar de los trabajadores, logrando de esta manera ahorros importantes en el consumo de agua y energía (Reyes y Galván, 2009). En la medida en que se proteja el ambiente inmediato, se pueden conservar los recursos naturales y garantizar un legando de supervivencia para las futuras generaciones (Prolys, 2008; Galván y Reyes, 2009).

En este contexto, los trabajadores entrevistados perciben que no existe una campaña ambiental efectiva por parte de la empresa. Hasta la fecha, esta campaña se viene realizando a través de la intranet y si el trabajador no dispone de un computador no tendrá acceso a la información, es decir, la campaña ambiental no se está realizando de forma efectiva para todo el personal.

En relación con la participación en diferentes actividades ambientales, los trabajadores manifestaron su disposición a participar en talleres de educación ambiental (40\%), en proyectos de mejoras ambientales para la planta de producción (44\%) y en trabajos con la comunidad (10\%).

Los talleres ambientales bien planificados deben estar enfocados en la prevención y el control de la contaminación que causa el proceso productivo de la empresa, por lo tanto la incorporación de las buenas prácticas ambientales y el cumplimiento de la legislación deben ser incluidos en estas actividades ya que apuntan hacia los principios básicos del Desarrollo Sustentable (Gabaldón, 2006). Cuando se hace referencia a talleres ambientales bien estructurados se pretende fundamentar los principios básicos de la Educación Ambiental (UNESCO/PNUMA, 1987). En este contexto, la Educación Ambiental se ha convertido en una estrategia para fomentar el desarrollo de una cultura de valoración y respecto hacia el medio ambiente e incentivar la participación activa de la ciudadanía en la gestión ambiental. Una vez implementado y consolidado un programa de Educación Ambiental por parte de la empresa será posible dar cabida a otras acciones que fomenten la valoración del ambiente como un patrimonio natural y cultural, enfocado hacia la comunidad para participar y cooperar activamente de forma conjunta (Reyes y col., 2002; Ochoa y col., 2014). 
Los resultados de las entrevistas muestran que existe poca receptividad de los trabajadores hacia la participación en trabajos con la comunidad cercana a la empresa. Esta percepción responde a la idea de que esta actividad no es su responsabilidad y que no cuentan con la información vinculante. En efecto, el trabajo con las comunidades requiere realizar previamente (Caride, 2001):

- Diagnóstico ambiental y sociocultural del área de estudio

- Diseño e implementación del Programa de Educación Ambiental

- Disponer del Recurso humano capacitado y soporte económico

- Evaluación del programa

Este tipo de proyecto requiere que las comunidades se encuentren organizadas e identifiquen los problemas ambientales de su entorno, trabajando de forma conjunta con la empresa para solventarlos.

\section{Responsabilidad Social Empresarial (RSE)}

Los resultados obtenidos indican que la mayoría de los entrevistados, conocen los términos de Responsabilidad Social Empresarial. Esto podría facilitar la implementación de una propuesta de RSE enfocado hacia el ambiente. La RSE estaría basada en la prevención, el control primario en la fuente, el enfoque sistemático, la reducción de la generación de materiales peligrosos y no peligrosos, el manejo integral y la planeación estratégica.

La mayoría de los trabajadores entrevistados (71\%) perciben que la empresa no realiza trabajos de RSE. Esta percepción parece ajustarse a la realidad, porque la empresa sólo realiza actividades enmarcadas en:

- Operativos de venta de cemento a la comunidad

- Operativos de venta de rubros que comprenden la cesta básica

- Cursos de capacitación a los trabajadores como: programas de Excel y otros

- Trabajo voluntario de los trabajadores internamente para recuperar algún área de la instalación

- Jornadas deportivas

- Jornadas de vacunación al personal de Planta.

Revisando estas actividades se puede observar que el enfoque de la empresa es meramente filantrópico. En la actualidad la RSE promueve una nueva forma de gestión de la empresa, más allá de acciones sociales o de la filantropía. Se trata de una manera de actuar que significa una mejor y eficaz interrelación entre accionistas, clientes, consumidores, proveedores y trabajadores; y una mejora del funcionamiento interno de las empresas, con especial referencia a los Consejos de Administración y a los aspectos vinculados a las relaciones laborables, en particular los relativos a la igualdad, al estímulo en la formación especializada continua de los trabajadores, el establecimiento de planes sociales y a la prevención de los riesgos laborales. Se trata de un 
enfoque que se basa en un conjunto integral de políticas, actuaciones y programas centrados en el respeto por la ética, las personas, los aspectos sociales y el medio ambiente (Estrada, 2005; NORMA ISO 26.000:2010; Lara, 2012).

\section{Conclusiones y Recomendaciones}

A través de la identificación de los impactos ambientales generados por el proceso de producción del concreto premezclado, se pudo determinar que el aspecto más relevante y visible son las emisiones de polvo; por ello es importante apuntar todos los esfuerzos en prevenir, mitigar y corregir dichas emisiones mediante mejoras tecnológicas.

A partir de las entrevistas se pudo determinar que es conveniente la elaboración de un Programa de Educación Ambiental, ya que los trabajadores manifestaron su interés en este sentido. La esencia de este programa de Educación Ambiental debería contemplar el cambio de actitud de los trabajadores, en relación a una toma de conciencia sobre la importancia de conservar el ambiente y mejorar la calidad de vida.

Disponer de una Planta de Concreto premezclado obliga a la empresa a llevar un control estricto en cuanto a los impactos adversos significativos que causa la Planta a su entorno y a sus trabajadores, por ello es importante mantener una buena relación con los vecinos que vaya más allá del hecho de proveer concreto para la fabricación de viviendas y la construcción de vías y calles; su imagen corporativa ante la comunidad debe ser más activa en cuanto a su interés en lo ambiental y lo social.

En Venezuela existe el marco normativo que regula la materia de la RSE (Norma ISO 26000:2010) el cual permitiría implementar un Programa de Responsabilidad Social Empresarial y Gestión Ambiental integrado, tanto en las empresas públicas como privadas, con repercusiones positivas en su buen desempeño, fortaleciendo la confianza, mejorando la reputación y generando valor (Lara, 2012).

\section{Bibliografía}

Arias F. (2012). El Proyecto de Investigación. Sexta edición. Caracas: Episteme.

Caride J. (2001). La Educación Ambiental en el desarrollo humano: horizontes para la sustentabilidad ecológica y la responsabilidad social. Segovia: Centro Nacional de Educación Ambiental-CENEAM.

Comisión Nacional del Medio Ambiente (CONAMA). (2001). Guía para el Control y la Prevención de la Contaminación Industrial. Rubro Productos de Cementos y Hormigón. Chile. 
Constitución de la República Bolivariana de Venezuela (2000). Gaceta Oficial N 5.453 / G.O. 5.908 E del 19-2-2009.

Clemente A, Souza A, Galván L y Reyes R. (2005). Estrategias empresariales para la Conservación ambiental en el Sector Industrial. Universidad, Ciencia y Tecnología. 9(33): 3-9.

Decreto № 2.217. (1992). Normas sobre el control de la contaminación generada por ruido, Publicado en Gaceta Oficial № 4.418 Extraordinario del 27-04-92.

Decreto № 2.216. (1992). Normas para el manejo de desechos sólidos de origen doméstico, comercial, industrial o de cualquier otra naturaleza que no sean peligrosos, publicado en la Gaceta Oficial № 4.418 Extraordinario del 27-04-92.

Decreto $N^{\circ}$ 883. (1995). Normas para la clasificación y el control de la calidad de los cuerpos de agua y vertidos o efluentes líquidos. Gaceta oficial № 5.021 Extraordinario, de fecha 18-12- 95.

Decreto № 638. (1995). Normas sobre calidad del aire y control de la contaminación atmosférica, publicado en Gaceta Oficial № 4.899 Extraordinario del 19-04-95.

Decreto № 1.257 (1996). Normas sobre evaluación ambiental de actividades susceptibles de degradar el ambiente, publicado en Gaceta Oficial № 35.946 del 25-04-96.

Decreto $N^{\circ}$ 2.673. (1998). Normas sobre emisiones de fuentes móviles. Gaceta Oficial №36.532 de fecha 04-09-98.

Decreto № 2.635. (1998). Normas para el control de la recuperación de materiales peligrosos y el Manejo de desechos peligrosos, publicado en la Gaceta Oficial № 5.245 Extraordinario del 03-08-98.

Estrada J. (2005). Ética, Responsabilidad Social Empresarial y Desarrollo Sustentable en las Organizaciones. Revista Administración y Organizaciones. 2005, Año 8, No. 15, pp. 27-45. 
Fonseca M y Reyes R. (2015). El concreto. Proceso productivo e impacto al ambiente. En: Una visión general de la investigación en la Facultad de Arquitectura y Diseño de la Universidad de los Andes. Mérida, Venezuela: Publicaciones ULA (FADULA).

Gabaldón A. (2006). Desarrollo Sustentable. La salida de América Latina. Primera Edición. Barcelona:Grijalbo.

Galán, I. (2010). Estudio del efecto sumidero de C02 de los materiales de base cemento. Revista Técnica Cemento Hormigón, No. 939. Consultado el 12 de Marzo de 2014. Disponible en: http://oa.upm.es/8747/

Galván L y Reyes R. (2009). Algunas herramientas para la prevención, control y mitigación de la contaminación ambiental. Universidad, Ciencia y Tecnología. 13(53): 287-294.

Galván L, Clemente A y Reyes R. (2012). Diagnóstico ambiental del sector industrial de Paraná, Brasil. Universidad, Ciencia y Tecnología.16(64): 76-84.

García, N. (2004). Manifestación de impacto ambiental. Extracción y trituración de roca caliza. México: Grupo Calider SA de CV.

Guédez C, de Armas D, Reyes R y Galván L. (2003). Los sistemas de gestión ambiental en la industria petrolera internacional. Interciencia. 28(9): 528-533.

Hernández, R., Fernández, C \& Baptista, P. (2010). Metodología de la investigación (5ta. Edición). México, DF: McGraw-Hill/Interamericana Editores, SA de CV.

Lara, J. (2012). La Responsabilidad Social Empresarial. El camino para la organización innovadora, competitiva y sostenible. Memorias del Congreso Internacional RSE. Caracas: Universidad Católica Andrés Bello.

Ley sobre sustancias, materiales y desechos peligrosos. (2001). Gaceta Oficial № 5.554 Extraordinario del 03-11-01.

Ley de Aguas. (2007). Gaceta Oficial № 38.595 del 02-01-07.

Ley de Gestión Integral de la Basura. (2010). Gaceta Oficial № 6.071Extraordinario del 30-12-10. 
Normas COVENIN 2385:2000. (2000). Concreto y Mortero. Agua de Mezclado. Requisitos. NORMA ISO 26.000: 2010. Guía de Responsabilidad Social Empresarial. 2010.

Ochoa A, Pellegrini N y Reyes R. (2014). Programa de Educación Ambiental: Herramientas para la sustentabilidad agroambiental. Revista de Investigación-UPEL. 81(38): 201-213.

O'Reilly, V., Bancrofft, R. y Ruiz, L. (2010). Las tecnologías del concreto en su ciclo de vida. Revista Concreto y Cemento, Investigación y Desarrollo.1(2): 42-47.

PROLYS, CA. (2008). Taller: Gestión Ambiental en la Industria del Concreto. Caracas.

Reyes R y Galván L. (2009). Preventing Environmental Pollution through Monitoring, Clean Technologies, Education, Economics and Management. En: Laboy-Nieves (Ed.). Environmental Management, Sustainable Development and Human Health. New York: Taylor and Francis Publishers.

Reyes R, Galván, L, Guédez C y de Armas D. (2002). La Gerencia Ambiental en el sistema Productivo venezolano. Universidad, Ciencia y Tecnología. 6(23): 155-159.

Reyes R, Pellegrini N y Farah D. (2003). La educación ambiental para la pequeña minería aurífera en la reserva forestal Imataca, Estado Bolívar, Venezuela. Universidad, Ciencia y Tecnología. 7(28): 262-266.

UNESCO/PNUMA. Congreso Internacional UNESCO-PNUMA sobre la Educación y la Formación Ambientales. Divulga 1987. Consultado el 12 de marzo 2014. Disponible en: http://unesdoc.unesco.org/images/0007/000750/075072sb.pdf

Villegas A, Reyes R y Galván L. (2004). Problemática ambiental en Venezuela y el mundo. Universidad, Ciencia y Tecnología. 8(30): 117-125.

Villegas A, Reyes R y Galván L. (2005). Gestión ambiental bajo ISO 14001 en Venezuela. Universidad, Ciencia y Tecnología. 10(34): 63-69. 